\title{
Nonlinear Dynamical Multi-Scale Model of Associative Memory
}

\author{
Alexander M. Duda \& Stephen E. Levinson \\ Department of Electrical and Computer Engineering \\ University of Illinois at Urbana-Champaign \\ Urbana, USA \\ e-mail: amduda@illinois.edu \& selevins@illinois.edu
}

\begin{abstract}
How can we get such reliable behavior from the mind when the brain is made up of such unreliable elements as neurons? We propose that the answer is related to the emergence of stable brain states and we offer a model that illustrates how such states could arise. We discuss a new ab initio nonlinear dynamical multi-scale model that will serve as the foundation for an associative memory. Scale 0 consists of spiking HodgkinHuxley (HH) neurons. Scale 1 consists of components that are made up of large populations of $\mathrm{HH}$ neurons whose topological structure evolves according to a Hebbian-plasticity rule based on synchronous firing. The component's state is captured by the variance of phase synchrony for the population. Many such components are sparsely connected to form a large network, whose state can be captured by the n-tuple consisting of the individual states of each member component. Scale 2 takes the state of the overall network and upon examining the particular interrelationships of each component (determining how the state of one component affects the state of others) is able to generate a class of trajectories that is multistationary and stable periodic. Such a class we consider a memory; the encoding of many such memories leads to the creation of a robust associative memory. The details of the different scales are examined.

Index Terms-machine learning; associative memory; nonlinear dynamics; multi-scale modeling; attractors; Hodgkin-Huxley neurons
\end{abstract}

\section{INTRODUCTION}

Reality appears consistent and structured. When you see a red ball you know it is not a black cat and when you see a black cat you know it is not a red ball. Even if, as Plato contended, we are all merely seeing shadows upon the wall [1], when the shadow of a circle is seen it is not mistaken for the shadow of a square.

Since a very young age, naturally and with ease, we associate events that occur close in time and in space [2]. Large collections of such associations give rise to higher-order concepts which we carry with us and modify throughout our life. However, the brain, which clearly plays a central role in the development of such concepts, consists of a vast number of neurons that are known to be unreliable and noisy. How such stable concepts can arise from such unstable neurons is a great mystery, which we hope to understand a bit better through the development of our new model.

We present the beginnings of a new multi-scale model that will serve as the foundation for an associative memory, which we believe is the mechanism for general intelligence. This model includes a number of biophysically realistic design elements. However, our focus is on capturing the functionality of associative memory as it works in humans (not necessarily modeling all the details found in the neocortex). To this end, we have introduced methods that have thus far not been used in this manner.

We believe that our model will enable a robust way of encoding noisy, complex, multi-dimensional real-time input streams from the external world (speech, visual scenes, etc.) into a low-dimensional trajectory form. At the very least, we hope to contribute another perspective on how to achieve intelligent behavior that evolves in a manner similar to that of humans.

In Section II we examine Scale 0, which consists of Hodgkin-Huxley neurons. We review the details of this canonical, conductance-based, spiking neuron model and provide an example of its nonlinear response, as well as its usefulness. In Section III we examine Scale 1, which is the component level. Here we discuss the causal Hebbian-plasticity rule that dictates the strength of connection between adjacent neurons, as well as the dynamic topological structure of the neuronal populations. Furthermore, we explain the method used to characterize the state of a given component (via phase synchrony). In Section IV we examine Scale 2, which is the memory encoding and recall level, where we use the time-varying states of components to generate classes of trajectories that may serve as memories. We explain the method used to encode such memories via an example. In Section V we discuss the application of this model to the intelligent behavior of a humanoid robot.

\section{Scale 0: Hodgkin-HuXley Neuron Model}

\section{A. Theoretical Background}

The model begins with the classic Hodgkin-Huxley $(\mathrm{HH})$ model of a single neuron [3]. What makes the $\mathrm{HH}$ neuron model most useful is the wide range of nonlinear behaviors observed from various inputs. For a detailed summary of these behaviors (tonic spiking, resonator, integrator, etc.) and a comparison of the most widely-used neuron models (including the integrate-and-fire, resonate-and-fire, Izhikevich, and FitzHughNagumo), which justifies its use as a biophysically meaningful neuron model, see [4].

We assume that the axon carries three primary currents: $I_{K}$ (voltage-gated persistent $K^{+}$), $I_{N a}$ (voltage-gated transient 
$\mathrm{Na}^{+}$), and $I_{L}$ (Ohmic leak). With $C$ as the membrane capacitance and $I_{a p p}$ as the applied current, the complete set of space-clamped $\mathrm{HH}$ equations in standard form are as follows:

$$
\begin{aligned}
C \frac{d V}{d t}=I_{a p p} & -I_{K}-I_{N a}-I_{L} \\
\frac{d n}{d t} & =\frac{n_{\infty}(V)-n}{\tau_{n}(V)} \\
\frac{d m}{d t} & =\frac{m_{\infty}(V)-m}{\tau_{m}(V)} \\
\frac{d h}{d t} & =\frac{h_{\infty}(V)-h}{\tau_{h}(V)}
\end{aligned}
$$

Where we have:

$$
\begin{gathered}
I_{K}=\bar{g}_{K} n^{4}\left(V-E_{K}\right) \\
I_{N a}=\bar{g}_{N a} m^{3} h\left(V-E_{N a}\right) \\
I_{L}=g_{L}\left(V-E_{L}\right)
\end{gathered}
$$

and:

$$
\begin{gathered}
n_{\infty}(V)=\frac{\alpha_{n}}{\alpha_{n}+\beta_{n}}, \quad \tau_{n}(V)=\frac{1}{\alpha_{n}+\beta_{n}}, \\
m_{\infty}=\frac{\alpha_{m}}{\alpha_{m}+\beta_{m}}, \quad \tau_{m}(V)=\frac{1}{\alpha_{m}+\beta_{m}}, \\
h_{\infty}=\frac{\alpha_{h}}{\alpha_{h}+\beta_{h}}, \quad \tau_{h}=\frac{1}{\alpha_{h}+\beta_{h}} .
\end{gathered}
$$

The parameters $\left\{\alpha_{n}, \beta_{n}, \alpha_{m}, \beta_{m}, \alpha_{h}, \beta_{h}\right\}$ correspond to a membrane potential shift and are defined as follows:

$$
\begin{gathered}
\alpha_{n}(V)=0.01 \frac{10-V}{\exp \left(\frac{10-V}{10}\right)-1}, \quad \beta_{n}(V)=0.125 \exp \left(\frac{-V}{80}\right), \\
\alpha_{m}(V)=0.1 \frac{25-V}{\exp \left(\frac{25-V}{10}\right)-1}, \quad \beta_{m}(V)=4 \exp \left(\frac{-V}{18}\right), \\
\alpha_{h}(V)=0.07 \exp \left(\frac{-V}{20}\right), \quad \beta_{h}(V)=\frac{1}{\exp \left(\frac{30-V}{10}\right)+1}
\end{gathered}
$$

Reasonable maximal conductances are

$$
\bar{g}_{K}=36 \frac{\mathrm{mS}}{\mathrm{cm}^{2}}, \quad \bar{g}_{N a}=120 \frac{\mathrm{mS}}{\mathrm{cm}^{2}}, \quad g_{L}=0.3 \frac{\mathrm{mS}}{\mathrm{cm}^{2}} .
$$

Furthermore, the shifted Nernst equilibrium potentials are

$$
E_{K}=-12 \mathrm{mV}, \quad E_{N a}=120 \mathrm{mV}, \quad E_{L}=10.6 \mathrm{mV} .
$$

To be clear, for the sodium and potassium ion channels, there is a corresponding probability that the (in)/activation gates will be (in)/active. These probabilities are represented by $n$, $m$, and $h$ (they are also referred to as "gating variables"). $I_{K}$ consists of four activation gates which results in the $n^{4}$ term where $P\{\mathrm{~K}$ act. gate is act. $\}=n$. $I_{N a}$ consists of three activation gates where $P\{\mathrm{Na}$ act. gate is act. $\}=m$ and one inactivation gate where $P\{\mathrm{Na}$ inact. gate is inact. $\}=h$; hence the $\mathrm{m}^{3} h$ term. $I_{L}$ is carried mainly by $\mathrm{Cl}^{-}$ions. In the human neocortex, neurons consist of additional currents with varied activation and inactivation dynamics. For our purposes though, the classic model is adequate.

\section{B. Simulation Result}

To offer the reader an example of the response of an $\mathrm{HH}$ neuron to an applied current, we have included the results of a single-pulse input of amplitude $18.25 \frac{\mu A}{\mathrm{~cm}^{2}}$ (which was enough to initiate an action potential and was largely inspired by [5]) in Fig. 1. Clearly we observe that the membrane begins at its rest voltage ( 0 millivolts). As the pulse arrives, the membrane quickly shows a large depolarization, which is followed by a steep upstroke. The membrane reaches a maximum of about 110 millivolts before it repolarizes, which takes about 1.5 milliseconds. One important feature of the model that distinguishes it from simpler models is the absolute and relative refractory periods, which capture the naturally observed resting periods where the neuron is either unable or unlikely to produce an action potential (if provoked by another pulse).

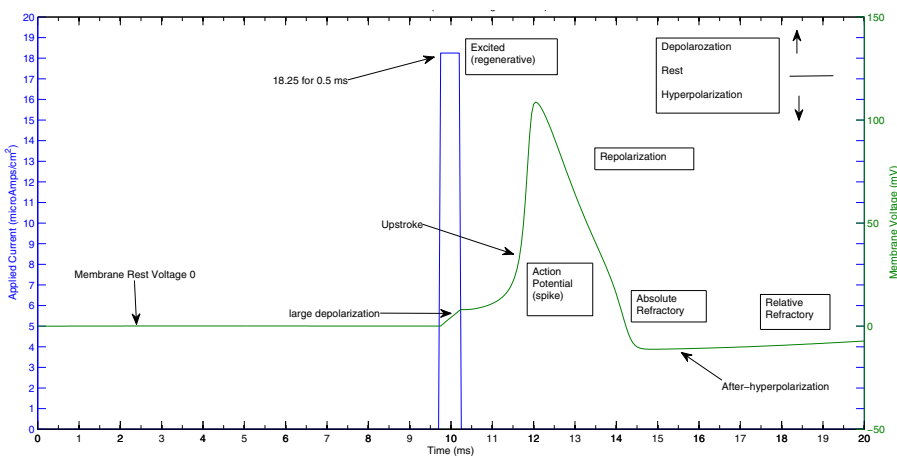

Fig. 1. Hodgkin-Huxley Neuron Model Membrane Response to a SinglePulse Input [5]

The interested reader should see [4] for a discussion on many of the behaviors observed from HH-type neurons.

\section{SCAlE 1: COMPONENTS}

As previously mentioned, Scale 1 is the component level. These components will consist of large populations of $\mathrm{HH}$ neurons. Adjacent ${ }^{1}$ neurons will obey a Hebbian plasticity rule [7], [8] based on causal synchronous spiking. The rule is illustrated in subsection $A$. The overall state of a component

\footnotetext{
${ }^{1}$ In the Graph Theoretic sense, two vertices are adjacent if they are joined by an edge [6].
} 
will be based on the variance of its phase synchrony. The scheme is illustrated in subsection $B$.

\section{A. Hebbian Plasticity Rule}

Suppose we have the structure shown in Fig. 2 whereby neuron four, labeled ' 4 ', has four neighbors: ${ }^{2}\{1,2,3,5\}$. Furthermore, suppose that each edge is bidirectional. ${ }^{3}$

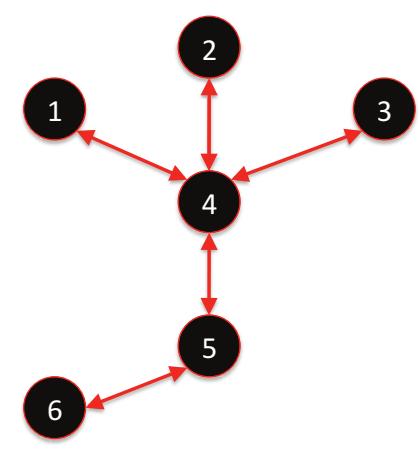

Fig. 2. Example Structure for Hebbian Plasticity Explanation

Let 4 spike. If one of its neighbors, $n \in\{1,2,3,5\}$, spikes within a short and biophysically meaningful period of time, $\tau$, then the strength of the edge $4 \rightarrow n$ will be increased. All neighbors that do not spike within $\tau$ will have the strength of the edge beginning at 4 decreased (this amount is smaller than the increase).

This computation is carried out for each such neuron and its neighborhood. Consequently, the edge weights will change based upon the synchronous spiking of neighboring neurons. The edge weights will determine the current distribution of a particular neuron to its neighbors upon spiking.

Considering that we also introduce a mechanism for neurons that are not neighbors to become neighbors with some probability, we can expect the network's topological structure to evolve over time.

\section{B. Phase Synchrony of Spiking Neurons}

Consider that a given neuron population will begin with some topological structure, whose adjacency relations may be generated with an Erdôs-Rényi Network Model [9]. For example, suppose we begin with 50 neurons and allow $\mathrm{p}=0.4$. Thus, between any pair of neurons there is a $40 \%$ chance of having a non-zero weighted edge. Obeying this rule will produce a graph structure like that shown in Fig. 3. Once the initial topology is set we may start hitting the network with applied current.

\footnotetext{
${ }^{2}$ Neighbors are the vertices adjacent to a given vertex [6]

${ }^{3}$ Each drawn edge can be considered two directed edges corresponding to the two different directions.
}

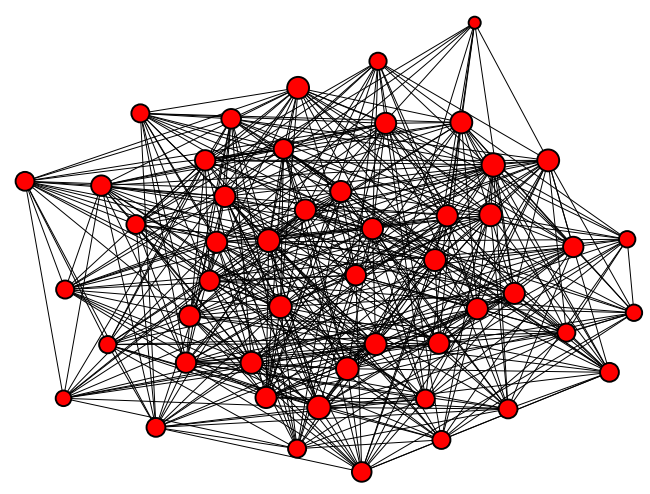

Fig. 3. Erdős-Rényi Network Model [9]. Example, N=50, p=0.4. (Note: For visual clarity, the size of each vertex is proportional to its degree.)
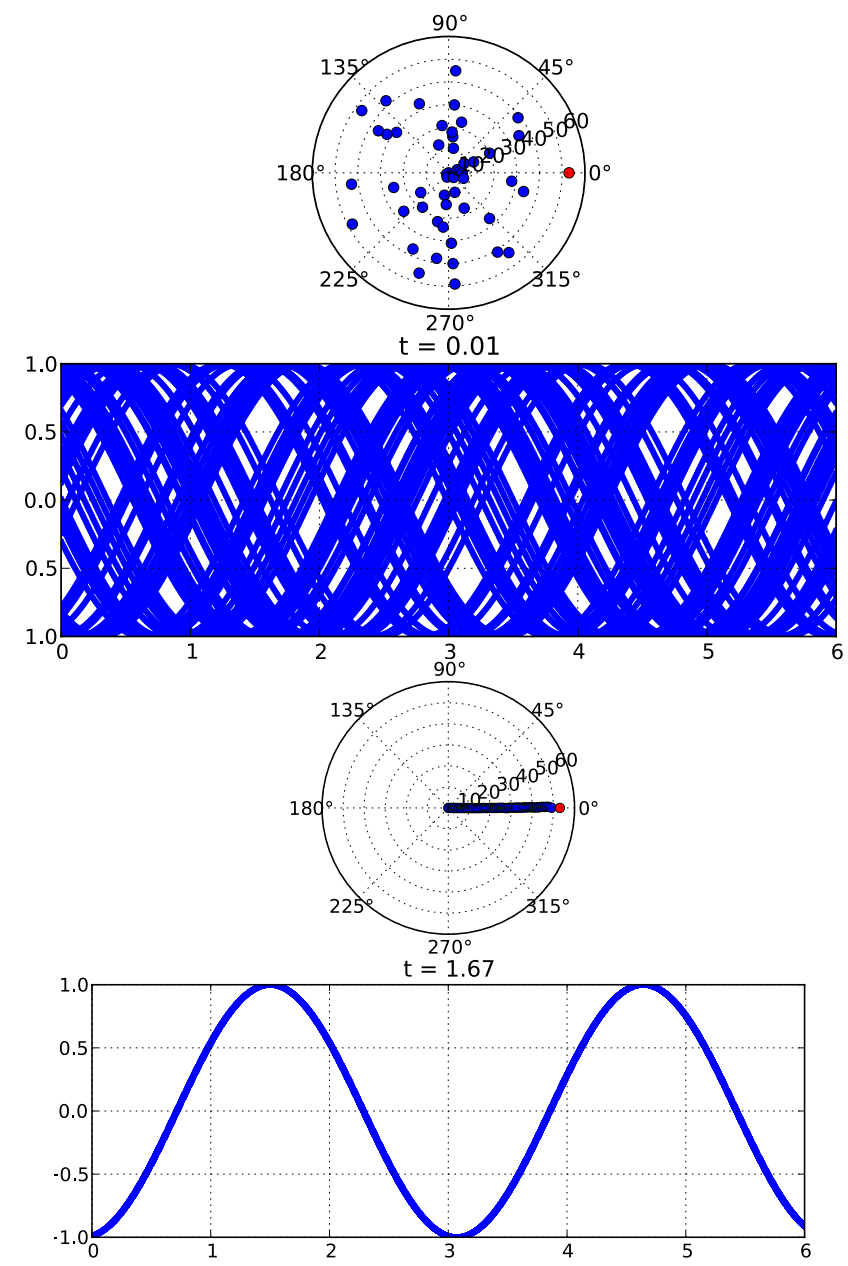

Fig. 4. Phase Synchrony at $\mathrm{t}=0.01,1.67 \mathrm{~s}$ for Erdôs-Rényi Network Model Example, $\mathrm{N}=50, \mathrm{p}=0.4$. (Note: Radial distance only exists to distinguish neurons.)

Assume that at the beginning each neuron has the same baseline firing rate [10], [11] (the rate at which the neuron fires before an external current is presented) but each is some degree out of phase with the mean of the population, which is made to represent 0 degrees on the unit circle in Fig. 4. 
Now, clearly from the $t=0.01$ seconds state of the neurons (which for the purposes of simplicity have been idealized as sinusoids), their phase angles are randomly sampled from the unit circle. However, after the input has been presented and 1.67 seconds has passed, we see that the neurons have begun to fire synchronously with negligible differences.

Given the high average degree ${ }^{4}$ of the network and the high edge density ${ }^{5}$, it is no surprise that moderately set initial edge weights would lead to a synchronized population. Allowing the edge weights to evolve continuously will allow the network topology to change in a realistic manner.
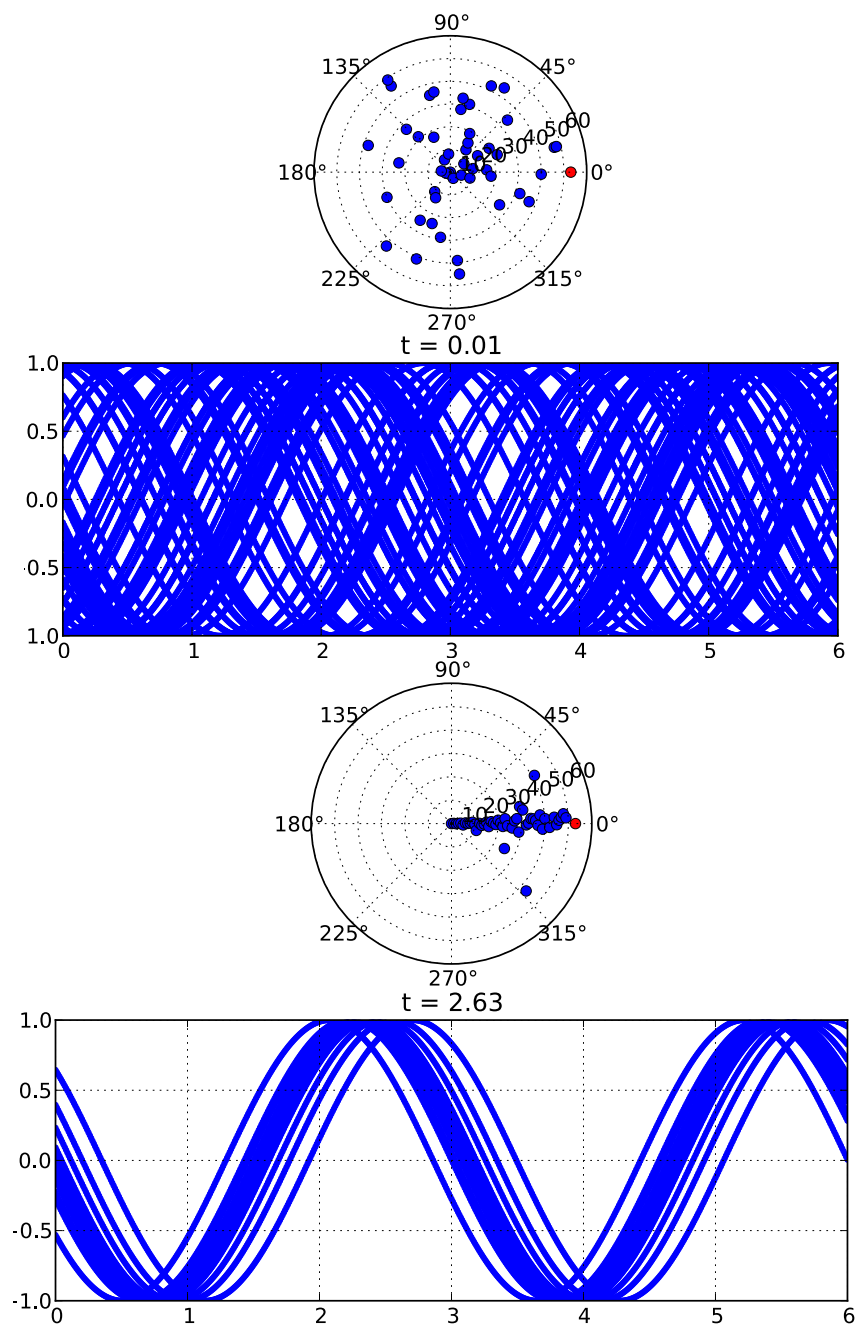

Fig. 5. Phase Synchrony at $\mathrm{t}=0.01,2.63 \mathrm{~s}$ for Barabási-Albert Scale-Free Network Model with $\mathrm{N}=50$. (Note: Radial distance only exists to distinguish neurons.)

Recent work has suggested that many real-world networks (including neural networks) converge to a Scale-Free network

\footnotetext{
${ }^{4}$ The degree of a vertex $v, d(v)$, is the number of edges incident to that vertex (when ignoring directionality) [6].

${ }^{5}$ Consider a given graph $G$ with $n$ vertices and $e(G)$ edges. Furthermore, recall that $K_{n}$ denotes the complete graph on $n$ vertices (every pair of vertices is adjacent) and has $e\left(K_{n}\right)$ edges [6]. The edge density of $G$ is then $\frac{e(G)}{e\left(K_{n}\right)}$.
}

structure $^{6}[12]-[14]$. Thus, it is reasonable to examine the phase synchrony dynamics of neurons on a canonical examplethe Barabási-Albert Scale-Free Network Model. In our example, the topology was iteratively developed until it consisted of 50 vertices that exhibited a power-law degree distribution (Fig. 6). Upon this structure neurons were placed as before. The level of phase synchrony was examined (Fig. 5). Clearly they do not synchronize perfectly. However, a tight angular region defines the boundaries for the phases. This can be accounted for by the structural property that in such networks, certain neurons emerge with very high degree, which means that those within their neighborhood will tend to synchronize. A number of such high degree neurons, which are clearly evident in Fig. 6, leads to the range of phase angles observed.

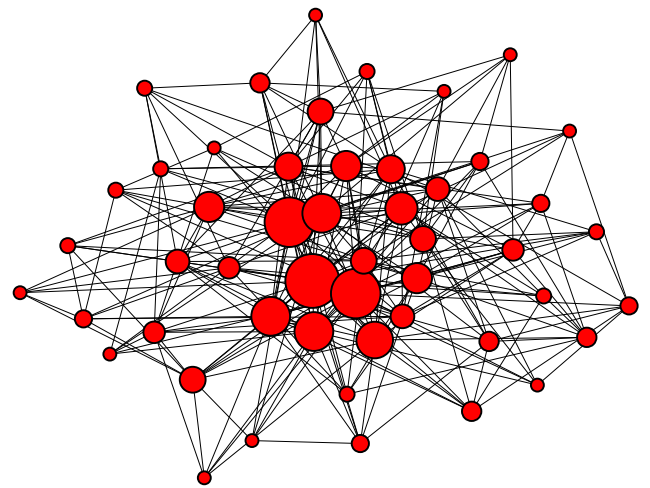

Fig. 6. Barabási-Albert Scale-Free Network Model [14] with N=50. (Note: For visual clarity, the size of each vertex is proportional to its degree.)

The state of the component can be characterized by the variance of the phase synchrony of the population, $\theta_{c}$. Using this metric we are able to take multi-dimensional, parallel input streams, feed them into a given population and map these to a 2D representation. The state of the different components along with information about how the components interrelate, can be used to uniquely determine a class of trajectories (deemed a memory), which is explained in the next section.

\section{Scale 2: Memory Encoding ANd ReCAll}

Let us suppose that we have $n$ components so that the system's state may be captured by the $n$-tuple: $\bar{\theta}=$ $\left(\theta_{1}, \theta_{2}, \ldots, \theta_{n}\right)$. Furthermore, assume that the components are sparsely connected, which means that there are few edges between components (neurons in different components are rarely adjacent). However, assume that there are enough such neighbors to allow current to pass between components and enable the phase synchrony of one component to affect the phase synchrony of another component. To measure these relationships we recall that with $\frac{d \theta}{d t}=\dot{\theta}$, the term $\frac{\partial \dot{\theta}_{i}}{\partial \theta_{j}}$ effectively represents the impact that component $j$ has on the time-rate-of-change of the phase synchrony of component $i$.

\footnotetext{
${ }^{6}$ In a Scale-Free network structure the probability that a given vertex has degree $\mathrm{k}$ follows a power-law distribution [12].
} 
We may use such terms to generate a set of ODEs that define all acceptable trajectories with such interrelationships. For clarity, allowing $a_{i j}=\frac{\partial \dot{\theta}_{i}}{\partial \theta_{j}}$, the set of ODEs may be written as follows:

$$
\begin{aligned}
\dot{\theta_{1}} & =a_{11} \theta_{1}+a_{12} \theta_{2}+\cdots+a_{1 n} \theta_{n} \\
\dot{\theta_{2}} & =a_{21} \theta_{1}+a_{22} \theta_{2}+\cdots+a_{2 n} \theta_{n} \\
\vdots & =\vdots \\
\dot{\theta_{n}} & =a_{n 1} \theta_{1}+a_{n 2} \theta_{2}+\cdots+a_{n n} \theta_{n}
\end{aligned}
$$

We are interested in those systems of ODEs that define multistationary and stable periodic trajectories. Thus, we must assert restrictions on the types of interrelationships among the components. The methods found in [15], [16] help us to achieve the goal. First let us consider the general 3component digraph shown in Fig. 7, whose vertex set consists of components and whose edge set consists of $a_{i j}$. We may examine the cycles to determine the proper restrictions. Within the framework discussed in [15], [16] there are two types of cycles: positive and negative. Positive cycles are those cycles such that the product of their edge weights are positive. Similarly, negative cycles are those cycles such that the product of their edge weights are negative. Positive cycles ensure multistationarity (with or without oscillations) while negative cycles ensure stable periodicity. Thus, for our purposes, a given graph must contain both positive and negative cycles to produce the desired memories.

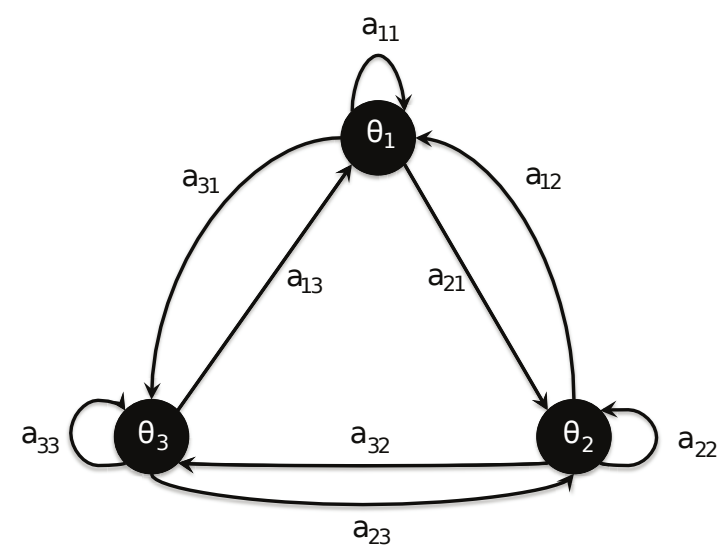

Fig. 7. Digraph example: 3 components with labeled edge weights

Now that we have defined the kinds of interrelationships that must exist among components (which can be quickly verified by checking the cycles within their corresponding digraph), we discuss the process of encoding such memories.

\section{A. Encoding}

Consider bombarding some subset of neurons with current. This will perturb the system and cause $\bar{\theta}$ to follow a specified trajectory in phase space. While this particular trajectory is traversed, one may determine the $a_{i j}$ of the system empirically.
These coefficients may be said to define a class of trajectories of which the observed trajectory is but a member. In this sense, the class of trajectories may be viewed as a memory. Any number of such memories may be encoded.

\section{B. Recall}

Consider after encoding, bombarding some subset of neurons with current again. If the particular current causes the $\bar{\theta}$ to follow a trajectory that is within one of the specified classes (a trajectory contained in one of the encoded memories), then it will be associated with that memory. This method could be suitable for properly associating complete, partial, or corrupted inputs with their true memory. Furthermore, the class of trajectories for a given memory could be thought of as a means of generalizing and associating similar inputs. The presence of multiple memories can be thought of as a means of classifying inputs. The method could be used for inputs originating externally or internally.

\section{Example.}

To be clear, let us examine a toy example where $n=3$ and the coefficients have been empirically determined:

$$
\begin{array}{lccc}
a_{11}=\frac{\partial \dot{\theta}_{1}}{\partial \theta_{1}}=0.5 & a_{12}=\frac{\partial \dot{\theta}_{1}}{\partial \theta_{2}}=0 & a_{13}=\frac{\partial \dot{\theta}_{1}}{\partial \theta_{3}}=-1 \\
a_{21}=\frac{\partial \theta_{2}}{\partial \theta_{1}}=-1 & a_{22}=\frac{\partial \theta_{2}}{\partial \theta_{2}}=-1 & a_{23}=\frac{\partial \theta_{2}}{\partial \theta_{3}}=0 \\
a_{31}=\frac{\partial \dot{\theta}_{3}}{\partial \theta_{1}}=3 \theta_{1}^{2} & a_{32}=\frac{\partial \theta_{3}}{\partial \theta_{2}}=1 & a_{33}=\frac{\partial \theta_{3}}{\partial \theta_{3}}=0
\end{array}
$$

As a result, the system may be captured with the following set of equations:

$$
\begin{aligned}
& \dot{\theta_{1}}=0.5 \theta_{1}-\theta_{3} \\
& \dot{\theta_{2}}=-\theta_{1}-\theta_{2} \\
& \dot{\theta_{3}}=3 \theta_{1}^{2}+\theta_{2}
\end{aligned}
$$

Furthermore, the system may be visualized with the following digraph $D$ :

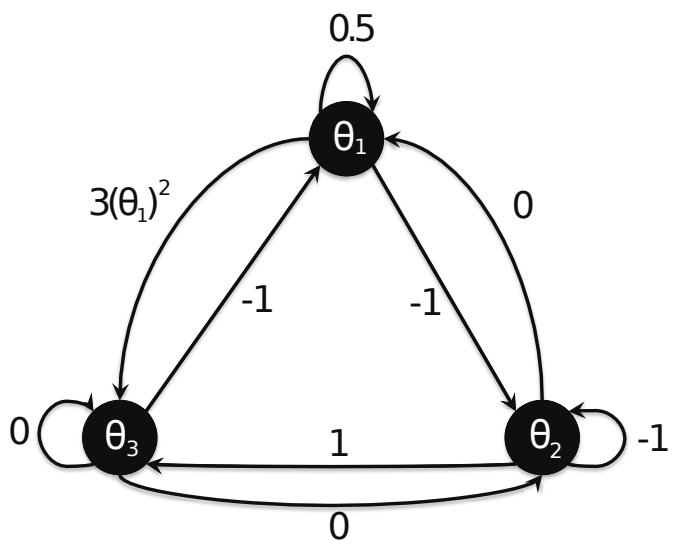

Fig. 8. Toy example: digraph D with 3 components and simple edge weights

This representation makes it clear the effect one component has on another in response to the stimuli. By simply examining 
the cycles in D, which are shown in Fig. 8, we can determine qualitative features about the corresponding trajectories, as discussed previously.

Clearly, D has a positive 3-cycle $\left(\theta_{1} \rightarrow \theta_{2} \rightarrow \theta_{3}\right)$ and a negative 2-cycle $\left(\theta_{1} \rightarrow \theta_{3}\right)$. As a result, we know that it will be of the proper type, according to the criteria previously explained (it will feature multiple stationary states and stable periodicity). To be sure, by examining Fig. 9, the class of trajectories comprising the memory is evident. This memory could be encoded into the system so that when similar trajectories result from systemic perturbations, the system's trajectory will be associated with the corresponding memory.

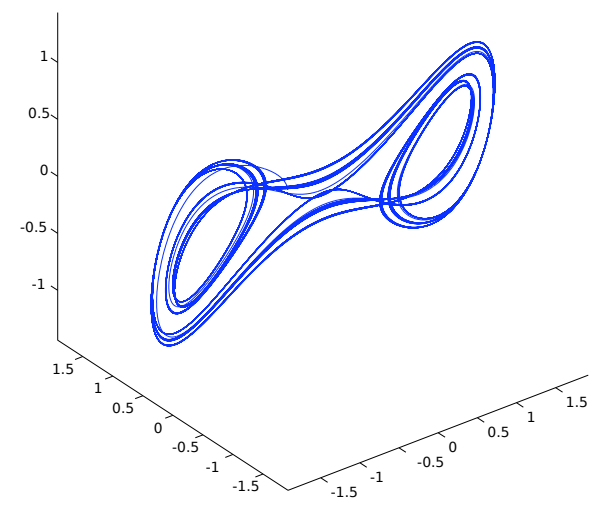

Fig. 9. Toy example memory representing a class of associated trajectories

\section{CONCLUSION}

This model enables a robust way of encoding noisy, complex, multi-dimensional real-time input streams from the external world (speech, visual scenes, etc.) into a low-dimensional trajectory form. Due to the hierarchical structure, the nature of the networked $\mathrm{HH}$ neurons, the metric used to characterize the components state, and the memory encoding and recall method, we anticipate that our model will be extremely robust even when presented with partial inputs or failing neuronal subnetworks. We have integrated aspects that are biophysically plausible but have focused on capturing the functionality of associative memory as it works in humans and thus have introduced methods that have hitherto been unused in this manner. At the very least, we hope to contribute another perspective on how to achieve intelligent behavior that evolves in a manner similar to that of humans.

In the near-term we hope to fully implement the model using many $\mathrm{HH}$ neurons per component and at least 3 components (we will restrict the component number in order to aid the dimensionality reduction). This computationally intensive work will be carried out on the Turing Cluster [17] or Blue Waters Supercomputer [18] (once it goes online). Furthermore, we will implement the model within Bert, our humanoid iCub [19] robot, which will allow the testing of our model in an embodied framework. To learn more about the group's activities with Bert, please see [20], [21] which outlines a number of the projects carried out by the Language
Acquisition and Robotics Group at the Beckman Institute for Advanced Science and Technology at the University of Illinois at Urbana-Champaign.

\section{ACKNOWLEDGMENT}

The authors would like to thank Dr. Fredrick Rothganger, Senior Member of Technical Staff at Sandia National Laboratories for his thoughtful discussions and strong advocacy of the project. Furthermore, the authors would like to acknowledge the generous support provided by Sandia National Laboratories, which enabled our progress. Finally, the authors would like to thank the anonymous reviewers of this paper, for their helpful suggestions. The first author would also like to thank the other members of the Language Acquisition and Robotics Group (Lydia Majure, Logan Niehaus, Aaron Silver, and Luke Wendt) for their stimulating conversations and enthusiasm about the model.

\section{REFERENCES}

[1] Plato, Republic (Oxford World's Classics translated by Robin Waterfield). New York, N.Y.: Oxford University Press, 2008, pp. 227-250.

[2] K. M. Squire and S. E. Levinson, "H.m.m.-based semantic learning for a mobile robot," IEEE Trans. Evol. Comput., vol. 11, no. 2, pp. 321-346, 2007.

[3] A. L. Hodgkin and A. Huxley, "A quantitative description of membrane current and its application to conduction and excitation in nerve," $J$. Physiol., vol. 117, pp. 500-544, 1952.

[4] E. M. Izhikevich, "Which model to use for cortical spiking neurons?" IEEE Trans. on Neur. Nets, vol. 15, no. 5, pp. 1063-1070, 2004.

[5] —, Dynamical Systems in Neuroscience. Cambridge, MA: MIT Press, 2007.

[6] D. B. West, Introduction to Graph Theory, 2nd Ed. Upper Saddle River, New Jersey: Prentice Hall, 2000.

[7] D. O. Hebb, The Organization of Behavior. New York, NY: Wiley, 1949.

[8] E. R. Kandel, "Small systems of neurons," Sci. Am., vol. 241, no. 3, pp. 66-76, 1979.

[9] P. Erdős and A. Rényi, "On random graphs. i,” Pub. Math., vol. 6, pp. 290-297, 1959.

[10] E. Brown, J. Moehlis, and P. Holmes, "On the phase reduction and response dynamics of neural oscillator populations," Neur. Comp., vol. 16, pp. 673-715, 2004.

[11] E. Brown, J. Moehlis, P. Holmes, E. Clayton, J. Rajkowski, and G. Aston-Jones, "The influence of spike rate and stimulus duration on noradrenergic neurons," J. of Comp. Neur., vol. 17, pp. 13-29, 2004.

[12] A. Barrat and M. Weigt, "On the properties of small-world network models," Eur. Phys. J. B, vol. 13, pp. 547-560, 2000.

[13] L. A. N. Amaral, A. Scala, M. Barthelemy, and H. E. Stanley, "Classes of behavior of small-world networks," Pr. Nat. Ac. of Sci. (USA), vol. 97 pp. 11 149-11 152, 2000.

[14] A.-L. Barabási and R. Albert, "Emergence of scaling in random networks," Science, vol. 286, pp. 509-512, 1999.

[15] R. Thomas and M. Kaufman, "Conceptual tools for the integration of data," C. R. Bio., vol. 325, pp. 505-514, 2002.

[16] M. Kaufman and R. Thomas, "Emergence of complex behaviour from simple circuit structures," C. R. Bio., vol. 326, pp. 205-214, 2003.

[17] Turing Xserve Cluster, http://www.cse.illinois.edu/turing/.

[18] Blue Waters Petascale Supercomputer, http://www.ncsa.illinois.edu/BlueWaters/.

[19] Robot Cub Consortium, http://www.robotcub.org.

[20] L. Majure, L. Niehaus, A. M. Duda, A. Silver, L. Wendt, and S. E. Levinson, "Integrating language and motor function on a humanoid robot," 2010, to be published.

[21] Language Acquisition and Robotics Group Website, http://www.isle.illinois.edu/acquisition/. 